\title{
Two-Electron Quantum Dot: Singlet-Triplet Splitting
}

\author{
M.K. Elsaid, M. Al-Naafa and S. Zugail \\ Department of Physics, Faculty of Sciences, \\ University of Hail, Hail, Saudi Arabia \\ mkelsaid@uoh.edu.sa
}

\begin{abstract}
The low-lying energy levels of a two electron quantum dot are calculated. We solve the relative part Hamiltonian of the quantum dot presented in a magnetic field of arbitrary strength using shifted $1 / \mathrm{N}$ expansion method. The dependence of the singlet-triplet energy gap $(J)$ on the strength of the magnetic field has been displayed. Based on comparisons, the shifted method gives very good results against exact and variation methods.
\end{abstract}

Keywords: Quantum Dots; Magnetic Field; Singlet-Triplet Energy Splitting

\section{Introduction}

Quantum dots (QDs), or artificial atoms, have been the subject of intense research studies over the last years. This growing interest is motivated by the physical effects and the potential device applications. Different methods ${ }^{[1-32]}$ have been used to investigate the energy spectrum of the interacting electrons confined in a quantum dot in the presence of an applied magnetic field. Two-electron QD, although it is a rather simple to study, yet a lot of useful information can be extracted and thus it serves as an important step to understand larger structures. In addition to this, the 2e case is essential component for the preparation of entangled electronic states and in double quantum dots is the basic of the quantum gate proposal ${ }^{[28]}$. Since one of the most interesting features of electron correlation is the oscillation of the spin from singlet $(S=0)$ to triplet $(\mathrm{S}=1)$ and angular momenta structure in the ground state of the QD system in the presence of a magnetic field. The QD, in this case has the 
potential to work as a qubit of a quantum computer ${ }^{[30]}$. In this work we shall use the shifted $1 / \mathrm{N}$ expansion method to solve the relative Hamiltonian of 2e QD under the effect of an applied magnetic field. In addition, we show the dependence of the singlet-triplet energy gap $J=$ $E_{t}-E_{s}$ on the strength of the magnetic field. $E_{t}$ And $E_{s}$ are the triplet and singlet QD ground states, respectively. The computer results are tested against various works published very recently, by Dybalski and Hawrylak $^{[27]}$ and Kandemir ${ }^{[29]}$.

\section{The Hamiltonian Theory}

The effective-mass Hamiltonian for interacting two electrons confined in a quantum dot-helium by a parabolic potential in uniform magnetic field of strength $B$ is given as,

$$
H=\sum_{i=1}^{2}\left\{\frac{P_{i}^{2}}{2 m^{*}}+\frac{1}{2} m^{*}\left[\omega_{0}^{2}+\frac{\omega_{c}^{2}}{4}\right] r_{i}^{2}+\frac{\omega_{c}}{2} \hat{L}_{i_{z}}\right\}+\frac{e^{2}}{\kappa\left|\vec{r}_{2}-\vec{r}_{1}\right|}
$$

Where $\omega_{0}$ is the confining frequency and $\kappa$ is the dielectric constant for the GaAs medium. $\vec{r}_{2}$ and $\vec{r}_{1}$ describe the positions of the first and second electron in the xy-plane. $\omega_{c}=\frac{e B}{m^{*} c}$ is the cyclotron frequency and the symmetric gauge $\vec{A}_{i}=\frac{1}{2} \vec{B} \times \vec{r}_{i}$ is used in Eq.(1). Upon introducing the center-of-mass (cm) $\vec{R}=\frac{\vec{r}_{1}+\vec{r}_{2}}{2}$ and the relative coordinates $\vec{r}=\vec{r}_{1}-\vec{r}_{2}$, the Hamiltonian in Eq. (1) can be decoupled to a center-of-mass $\left(H_{R}\right)$ and relative $\left(H_{r}\right)$ parts. The $\mathrm{cm}$-part is a harmonic oscillator type with a wellknown eigenenergies:

$$
E_{c m}=\left(2 n_{c m}+\left|m_{c m}\right|+1\right) \hbar\left[\omega_{0}^{2}+\frac{\omega_{c}^{2}}{4}\right]^{1 / 2}+m_{c m} \frac{\hbar \omega_{c}}{2}
$$

Where $n_{c m}=0,1,2, \ldots$ and $m_{c m}=0 \pm 1, \pm 2, \ldots$

The main task in this work is to solve the relative Hamiltonian part,

$$
H_{r}=\frac{p^{2}}{2 \mu}+\frac{1}{4}\left[\omega_{0}^{2}+\frac{\omega_{c}^{2}}{4}\right] r^{2}+\frac{e^{2}}{\kappa|\vec{r}|}+m \frac{\hbar \omega_{c}}{2}
$$


By using the shifted 1/N expansion method. With the parabolic confining potential, the QD Hamiltonian decouples to $\mathrm{CM}$ and relative parts. In the far-infrared (FIR) magneto absorption spectra the electromagnetic waves couple to CM-part and the resulting absorption spectra gives only two simple dispersion relations as function of magnetic field and does not depend on the number of confined electrons in the quantum dot nor on the interaction between them ${ }^{[31]}$. The energy states of the total Hamiltonian are labeled by the $\mathrm{CM}$ and relative quantum numbers $\left|n_{c m} m_{c m} ; n_{r} m\right\rangle$. The steps to produce the eigenenergies by the shifted method are given in Refs. ${ }^{[24,33-35]}$ and will not be repeated here. Only the necessary expressions to compute the energies will be presented. The energy eigenvalues in powers of $1 / \bar{k}$ (up to third order) reads as,

$$
E_{n, m}=E_{0}+\frac{\bar{k}^{2}}{4 r_{0}}+\frac{1}{r_{0}^{2}}\left[\frac{(1-\alpha)(3-\alpha)}{4}+\alpha_{1}\right]+\frac{\alpha_{2}}{\bar{k} r_{0}^{2}}
$$

where

$$
E_{0}=\frac{1}{r_{0}}+\frac{1}{4}\left[\omega_{0}^{2}+\frac{\omega_{c}^{2}}{4}\right] r_{0}^{2}+m \frac{\omega_{c}}{2}
$$

$\alpha_{1}$ and $\alpha_{2}$ are parameters expressed in terms of $Q, \varpi$ and quantum numbers $n_{r}$ and $m$, given in Ref. [24]. $\bar{k}=N+2|m|-a$, where $\mathrm{N}$ is the spatial dimension, shift parameter $a=2-\left(2 n_{r}+1\right) \varpi$ and $\varpi\left[3+\frac{V^{\prime \prime}\left(r_{0}\right)}{V^{\prime}\left(r_{0}\right)}\right]^{1 / 2}$. The roots $r_{0}$ (where the effective potential has a minimum) are determined for particular quantum state $\left|n_{r} m\right\rangle, \omega_{0}$ and $\omega_{c}$ through the relation:

$$
\left[2 r_{0}^{3} V^{\prime}\left(r_{0}\right)\right]^{1 / 2}=Q^{1 / 2}=\bar{k}=(2+2|m|-a)
$$

After obtaining the roots $r_{0}$, the eigenenergies can be computed using Eq. (4). $n_{r}$ is the radial quantum number related to the principle (n) one by the standard relation: $n_{r}=n-|m|-1$. 


\section{Results and Discussion}

Our computed results for $2 e \mathrm{QD}$, are presented in Fig. $1 \& 2$ and Table 1. The material parameters for quantum dot made from GaAs: $\stackrel{*}{m}_{e}=0.067$ and dielectric constant $\kappa=12.5$. We obtain the effective Rydberg $\stackrel{*}{R}=5.83 \mathrm{meV}$ and effective Bohr radius $\stackrel{*}{a}=9.87 \mathrm{~nm}$. In Fig. 1 we have shown the dependence of relative energies, produced by the shifted method, on the magnetic field strength with confinement energy $\hbar \omega_{0}=\frac{2}{3} R^{*}$ and different angular momenta quantum numbers $\mathrm{m}=0,-1,-$ 2 and -3 . The figure clearly shows the singlet-triplet transitions in the ground state of the QD. For example while the angular momentum of the ground state changes from $\mathrm{m}=0$ to -1 at $\omega_{c} \approx 0.6,-1$ to -2 at $\omega_{c} \approx 2$ and -2 to -3 at $\omega_{c} \approx 3$ the spin also oscillates between singlet and triplet states. To test further, the accuracy of shifted method, we have compared in Table 1 our result against perturbation, variation $E^{A}$ and numerical (exact) ones given by Kandemir ${ }^{[29]}$. It is obvious from the table that the shifted method is in very good agreement with exact and variation methods while the perturbation method deviates from all the methods.

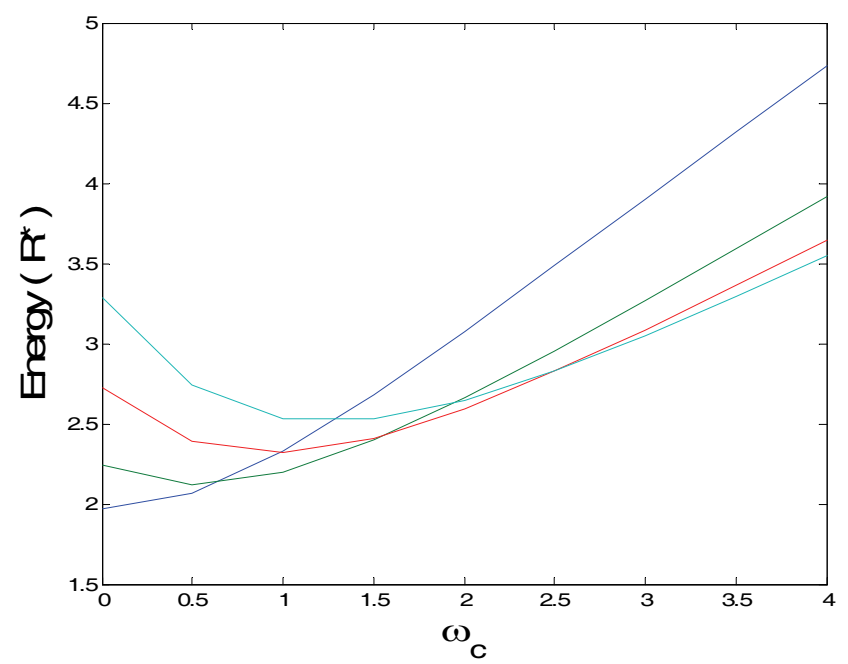

Fig. 1. The dependence of eigenenergies for two-electron quantum dot made from GaAs, on a magnetic field and various angular momentum $m=0,-1,-2$ and -3 . The confinement energy strength $\hbar \omega_{0}=\frac{2}{3} R^{*} .[\stackrel{*}{\boldsymbol{R}}=5.83 \mathrm{meV}$ and $\stackrel{*}{a}=9.87 \mathrm{~nm}]$. 
Table 1. The ground state energies (units of $\hbar \omega_{0}=11.857 \mathrm{meV}$ ) for quantum dot made from GaAs calculated by four different methods: perturbation $E_{0}^{p}$, analytical (variation) $E_{0}^{A}$ numerical $E_{0}^{N}$ and shifted $E_{0}^{1 / N}$ methods. The confining energy $\hbar \omega_{0}=3.32 \mathrm{meV}$, Ref. [27] . $[\stackrel{*}{R}=5.83 \mathrm{meV}$ and $\stackrel{*}{\alpha}=9.87 \mathrm{~nm}]$.

\begin{tabular}{|c|c|c|c|c|}
\hline $\mathbf{B}(\mathbf{T})$ & $\boldsymbol{E}_{0}^{\boldsymbol{p}}$ & $\boldsymbol{E}_{0}^{\boldsymbol{A}}$ & $\boldsymbol{E}_{0}^{\boldsymbol{N}}$ & $\boldsymbol{E}_{0}^{1 / \boldsymbol{N}}$ \\
\hline 0.0 & 1.22319 & 1.03223 & 1.02214 & 1.0354 \\
\hline 0.5 & 1.23071 & 1.03930 & 1.02928 & 1.0417 \\
\hline 1.0 & 1.25281 & 1.06012 & 1.05029 & 1.0605 \\
\hline 1.5 & 1.28831 & 1.03961 & 1.08408 & 1.0909 \\
\hline 2.0 & 1.33551 & 1.13821 & 1.12909 & 1.1310 \\
\hline 2.5 & 1.39252 & 1.19223 & 1.18360 & 1.1791 \\
\hline 3.0 & 1.45753 & 1.25396 & 1.24589 & 1.2341 \\
\hline 3.5 & 1.52890 & 1.32193 & 1.31446 & 1.2937 \\
\hline 4.0 & 1.60526 & 139485 & 1.38800 & 1.3576 \\
\hline 4.5 & 1.68551 & 1.47168 & 1.46547 & 1.4245 \\
\hline 5.0 & 1.76876 & 1.55158 & 1.54601 & 1.4934 \\
\hline
\end{tabular}

We have displayed, in Fig. 2, the energy gap $J\left(\omega_{c}\right)=E_{t}-E_{s}$ as a function of $\omega_{c}$ for confinement energy strength $\hbar \omega_{0}=\frac{2}{3} R^{*}$ calculated by shifted method. The figure is in agreement with both works given recently by Dybalkski and Hawrylak ${ }^{[27]}$ and Helle et al. ${ }^{[31]}$. However we have noticed a small qualitative difference between our $J$-curve and the corresponding one (Fig. 8), in Ref. ${ }^{[27]}$ For $\omega_{c} \sim 3.5$ range, our $J$-curve continues down and widening, while Fig. 8 in Ref. [27] shows a small kink and starts going up. In fact the behavior of $J$-curve is also calculated by Helle et al., in Ref. ${ }^{[31]}$, and is in agreement with our results. We can see also, from Fig. 1, that the triplet state $(\mathrm{m}=-3)$ is becoming lower than the singlet state $(\mathrm{m}=-2)$ and the energy gap, $J$, starts widening supporting our results. The figure shows a change in the sign of $J$ from positive to negative. This is a practical indication that $J$ can be tuned by varying an external magnetic field. 


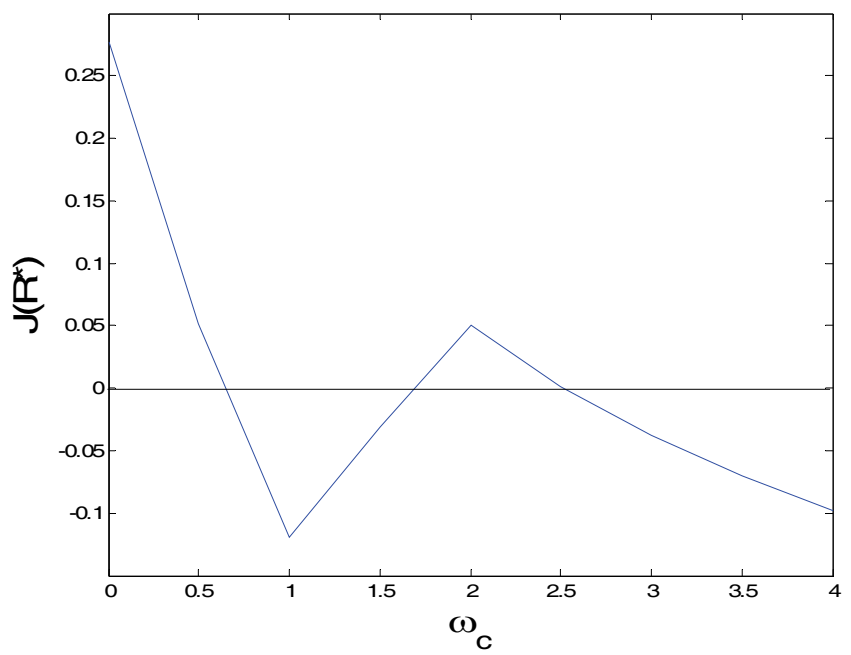

Fig. 2. The dependence of singlet-triplet energy gap, $J=E_{t}-E_{s}$ on the strength of the magnetic field $\omega_{c}$ for two-electron quantum dot made from GaAs. $[\stackrel{*}{R}=5.83 \mathrm{meV}$ and $\stackrel{*}{a}=9.87 \mathrm{~nm}]$.

\section{Conclusions}

In conclusion, we have studied the spectroscopic properties of the 2e QD presented in a magnetic field. We have computed the eigenenergies of the singlets and the triplets states of the QD system. The oscillations in the angular momentum (m) and spin (S) of the ground state against the magnetic field. We have plotted the dependence of the energy gap difference, $J=E_{t}-E_{s}$ as a function of magnetic field strength, $\omega_{c}$. Based on comparisons with exact and variational methods, the shifted method gives very good results for all ranges of magnetic field strength.

\section{References}

[ 1 ] Drexler, H., Leonard, D., Hansen, W., Kothaus, J.P. and Petroff, P.M., Phys. Rev. Lett. 73: 2252 (1994).

[ 2 ] Sikorski, C. and Merks, U., Phys. Rev. Lett., 62: 2164 (1989).

[ 3 ] Demel, T., Hietmann, D., Grambow, P. and Plog, K., Phys. Rev. Lett., 64: 788 (1990).

[ 4 ] Lorke, A., Kothaus, J.P. and Ploog, K., Phys. Rev. Lett., 64: 2259 (1990).

[ 5 ] Ashoori, R.C., Weiner, J.S., Pfeiffer, L.N., Baldwin, K.W. and West, K.W., Phys. Rev. Lett., 71: 613 (1993).

[ 6 ] Makysm, P.A. and Chakraborty, T.T., Phys. Rev. Lett., 65: 108 (1990).

[ 7 ] Wagner, M., Merkt, U. and Chapllik, A.V., Phys. Rev., B 45: 1951 (1992).

[ 8 ] Pfannkuche, D. and Gerharst, R.R., Physica, B 189: 6 (1993). 
[ 9 ] De Groote, J.J. S., Honos, J.E.M. and Chapllik, A.V., Phys. Rev., B 46: 12773 (1992).

[ 10 ] Merkt, U., Huser, J. and Wagner, M., Phys. Rev., B 43: 7320 (1991).

[11] Pfannkuche, D. and Gerhardts, R.R., Phys. Rev., B 44: 13132 (1991).

[ 12 ] Zhu, K.D. and Gu, S.W., Phys. Rev. Lett., A172: 296 (1993).

[ 13 ] Jonson, N.F. and Payne, M.C., Phys. Rev. Lett., 67: 1157 (1991).

[ 14 ] Klama, S. and Mishchenko, E.G., J. Phys Condens. Matter, 10: 601 (1998).

[ 15 ] Zhu, J.L., Li, Z.Q, Yu, J.Z., Ohno, K. and Kawazoe, Y., Phys. Rev., B 55: 15819 (1997).

[ 16 ] Dineykhan, M. and Nazmitdinov, R.G., Phys. Rev., B 55: 133707 (1997).

[ 17 ] Bryant, G., Phys. Rev. Lett., 59: 1140 (1987).

[ 18 ] Zhu, J.L., Kawazoe, Y. and Yao, T., J. Phys. Condens. Matter, 11: 299 (1999).

[19] Animsomovas, E. and Matulis, J. Phys. Condens. Matter, 10: 601 (1998).

[ 20 ] Matulis, A. and Peeters, P.M., J. Phys. Condens. Matter, 6: 775 (1994).

[ 21 ] Blanter, Y.M., Kaputkinaand, N.E. and Lozovik, Y.E., Physica Seripta, 54: 539 (1996).

[ 22 ] Peeters, F.M. and Schweigert, V.A., Phys. Rev., B 53: 1468 (1996).

[ 23 ] Oh, J.H., Hcang, K.J., Ihm, G. and Lee, S.J., Phys. Rev., B 53: 13264 (1996).

[ 24 ] El-Said, M., Semicond. Scien. Technol., 10: 1310 (1995).

[ 25 ] Nasmitdinov, R.G., Simonovic, N.S. and Rost, J.M., Phys. Rev., B 65: 155307 (2002).

[ 26 ] Drouvelis, P.S., Schmelcher, P. and Diakonos, F.K., Phys. Rev., B 69: 155312 (2004).

[ 27 ] Dybalski, W. and Hawrylak, P., Phys. Rev., B 72: 205432 (2005).

[ 28 ] Burkard, G., Loss, D. and Di Vincenzo, D.P., Phys. Rev., B59: 2070 (1999).

[ 29 ] Kandemir, B.S., Phys. Rev., B 72: 205432 (2005).

[ 30 ] Ciftja, O. and Faruk, M.G., Phys. Rev., B 72: 205334 (2005).

[ 31 ] Helle, M., Harju, A. and Nieminen, R.M., Phys. Rev., B 72: 205329 (2005); arXive:condmat/0511577.

[ 32 ] Harju, A., Siljamaki, S. and Nieminen, R.M., Phys.Rev.Lett., 88: 226804 (2002).

[ 33 ] Imbo, T., Pagnamento, A. and Sukhatme, Phys. Rev., D 29: 8763 (1984).

[ 34 ] Imbo, T. and Sukhatme, U., Phys. Rev., D 28: 418 (1983); ibid, 31: 2655 (1985).

[ 35 ] Mukherji, R.D. and Varnishni, Y.P., J. Phys., B 19: 3411 (1986). 


\section{نقطة كمية ذات إلكترونين: فصل مستويات الطاقة الأحادية و الثثلانية}

\section{محمد خليل السعيد ، ومحمد بن عبدالعزيز النافع ، وصلاح زقيل}

قسم الفيزياء ، كلية العلوم ، جامعة حائل، حائل ، المدلكة العربية السعودية mkelsaid@uoh.edu.sa

المستخلص. نم حساب مستويات الطاقة المنخفضة لنقطة كمية ذات إلكترونين. نم أيضًا حل الجزء النسبي من الهاملتونين (دالّة الطاقة)

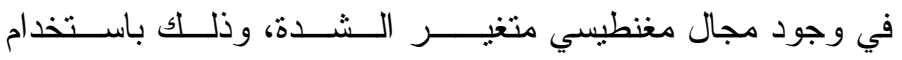
طريقــة مفكوك N N / المز اح. تم الحصول على مستويات الطاقة

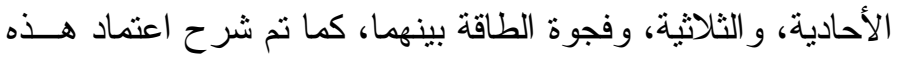

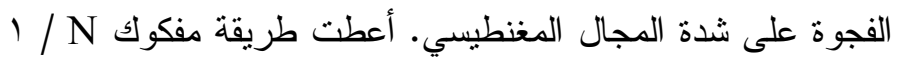

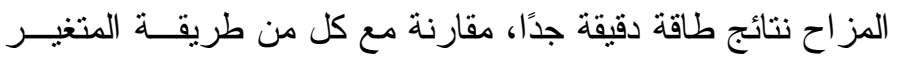
و الطرق الحسابية الدقيقة (exact methods). 